\title{
The INTERBALL-Tail ELECTRON experiment: initial results on the low-latitude boundary layer of the dawn magnetosphere
}

\author{
J.-A. Sauvaud ${ }^{1}$, P. Koperski ${ }^{1}$, T. Beutier ${ }^{1}$, H. Barthe ${ }^{1}$, C. Aoustin ${ }^{1}$, J. J. Thocaven ${ }^{1}$, J. Rouzaud ${ }^{1}$, E. Penou ${ }^{1}$ \\ O. Vaisberg ${ }^{2}$, N. Borodkova ${ }^{2}$ \\ ${ }^{1}$ Centre d'Etude Spatiale des Rayonnements, BP4346, F-31029, Toulouse, France \\ ${ }^{2}$ Space Research Institute, Profsoyuznaya 84/32, Moscow, Russia
}

Received: 13 May 1996 / Revised: 24 January 1997 / Accepted: 28 January 1997

\begin{abstract}
The Toulouse electron spectrometer flown on the Russian project INTERBALL-Tail performs electron measurements from 10 to $26000 \mathrm{eV}$ over a $4 \pi$ solid angle in a satellite rotation period. The INTERBALLTail probe was launched on 3 August 1995 together with a subsatellite into a $65^{\circ}$ inclination orbit with an apogee of about $30 R_{E}$. The INTERBALL mission also includes a polar spacecraft launched in August 1996 for correlated studies of the outer magnetosphere and of the auroral regions. We present new observations concerning the low-latitude boundary layers (LLBL) of the magnetosphere obtained near the dawn magnetic meridian. LLBL are encountered at the interface between two plasma regimes, the magnetosheath and the dayside extension of the plasma sheet. Unexpectedly, the radial extent of the region where LLBL electrons can be sporadically detected as plasma clouds can reach up to 5 $R_{E}$ inside the magnetopause. The LLBL core electrons have an average energy of the order of $100 \mathrm{eV}$ and are systematically field-aligned and counterstreaming. As a trend, the temperature of the LLBL electrons increases with decreasing distance to Earth. Along the satellite orbit, the apparent time of occurrence of LLBL electrons can vary from about 5 to $20 \mathrm{~min}$ from one pass to another. An initial first comparison between electronand magnetic-field measurements indicates that the LLBL clouds coincide with a strong increase in the magnetic field (by up to a factor of 2). The resulting strong magnetic field gradient can explain why the plasma-sheet electron flux in the $\mathrm{keV}$ range is strongly depressed in LLBL occurrence regions (up to a factor of $\sim 10)$. We also show that LLBL electron encounters are related to field-aligned current structures and that wide LLBL correspond to northward interplanetary magnetic field. Evidence for LLBL/plasma-sheet electron leakage into the magnetosheath during southward IMF is also presented.
\end{abstract}

\section{Introduction}

Over the course of a year, the INTERBALL-Tail satellite orbit plane drifts across the magnetosphere and the solar wind, thus sweeping the dayside solar wind and the main plasma domains of the outer magnetosphere; the Earth's bow shock, the magnetopause boundary, the low-latitude boundary layers (LLBL), the polar cusp, the plasma mantle and the plasma and neutral sheets. Measurements of the plasma distribution with full $4 \pi$ coverage are essential to characterize the main plasma domains. INTERBALL, together with the other satellites of the International Solar-Terrestrial Physics Program (ISTP), is expected to provide substantial advances in the field of magnetospheric research. Progress is expected mainly in the understanding of the processes which lead to solar plasma entry into the magnetosphere, to large-scale plasma convection, to solar-wind energy accumulation in the tail and to plasma instabilities which give rise to substorms. Within this program, the main INTERBALL-Tail satellite and its subsatellite are particularly devoted to the detailed study of the energy, momentum and mass transfer in critical regions of the solar wind-magnetosphere system (see Galeev et al., 1995).

In particular, initial results of the ELECTRON experiment aboard INTERBALL Tail provide a characterization of the LLBL near the dawn meridian. Since the discovery of this layer of magnetosheath plasma earthwards of the magnetopause (Eastman et al., 1976, 1985), many studies have focused on this region, considered to be important for the transport of energy, mass and momentum from the magnetosheath to the magnetosphere (e.g. Ogilvie et al., 1984; Traver et al., 1991; Fuselier et al., 1995). ISEE and AMPTE data have shown that the LLBL are characterized by electrons in the $100-\mathrm{eV}$ range counterstreaming along the local magnetic field. While many studies have focused on 
the formation of the near-magnetopause LLBL (e.g. Fuselier et al., 1995), little, if any, information is available on its radial extent across the dawn/dusk magnetosphere. The large-scale structure of this layer and its relationship with the plasma sheet clearly deserve further study. The dayside LLBL has been shown to correspond to significant antisunward plasma flow (e.g. Lundin et al., 1991). LLBL is thus the site of a MHD electric power generator because it lies on closed, northward-directed magnetic field lines of the magnetosphere. Estimates of the voltage drop across the LLBL vary strongly from author to author, ranging from several kV (Mozer, 1984) up to $10-15 \mathrm{kV}$ (Heikkila, 1986). More recently, Stasiewicz (1994) presented new mapping arguments from the Viking polar satellite data and the Tsyganenko (1989) magnetic-field model suggesting that the plasma flow inside the LLBL could usually provide the dominant contribution to the total polar-cap potential drop $(\sim 40 \mathrm{kV})$.

We present here direct evidence for the existence of the LLBL as a very large scale structure of the magnetosphere. Electron measurements show that the dawn LLBL, characterized by field-aligned counterstreaming electron beams with an energy of $\sim 100 \mathrm{eV}$, can be encountered quasi-periodically inside the magnetopause over a region with an apparent radial extent as large as $5 R_{E}$, i.e. $1 / 3$ of the distance from the Earth to the magnetopause in the dawn sector. The detection of the LLBL electron clouds coincides with a sharp increase in the magnetic field (by up to a factor of 2) and a strong decrease (by up to an order of magnitude) in $\mathrm{keV}$ plasma-sheet electrons. These first INTERBALL-Tail electron observations clearly favour the LLBL as an important source region for solar-wind plasma in the magnetosphere.

\section{The ELECTRON experiment}

To measure the solar-wind and magnetospheric electron distribution functions, the ELECTRON experiment selects incoming electrons according to their energy by electrostatic deflection in a symmetrical hemispherical analyser having a uniform angle-energy response and detects them with two microchannel plates and discrete anodes. The detector measures the electron distribution in phase space over the complete solid angle, in the energy range $10-26000 \mathrm{eV}$, in a satellite rotation period (Sauvaud et al., 1995).

\subsection{The analyser}

The analyser is a symmetrical spherical electrostatic analyser with a uniform $360^{\circ}$ disk-shaped field of view, good angular resolution and fine energy resolution (Carlson et al., 1982; Lin, 1989). Because particles are subjected to a central force, their trajectory is planar and there is a one-to-one correspondence between the incident direction of the particle and the curvilinear abscissa reached by the particle at the analyser output (Fig. 1). This symmetrical quadrisphere or "top-hat" geometry avoids the shortcomings of the conventional quadrispherical analyser, namely a limited polar-angle range and severely distorted response characteristics (Gosling et al., 1978). The top-hat geometry has now been successfully used on numerous sounding rockets as well as on the AMPTE/IRM and GIOTTO spacecraft (Paschmann et al., 1985; Rème et al., 1986).

The electrostatic analyser has three concentric spherical section elements: an inner hemisphere to which the positive deflection voltage is applied, an outer hemisphere (with radius $R+\Delta R$ ) with a circular hole at its zenith, and a small top-cap section (with radius $R+2 \Delta R$ ) which, together with the outer hemisphere, defines the entrance aperture (Fig. 1). Because of the satellite geometry, an unobstructed $360^{\circ}$ field of view rotating around an axis parallel to the viewing plane (in order to cover the $4 \pi$ solid angle in half a spin period) was not available. The analyser was thus mounted to view half a plane containing the satellite rotation axis. Thus, a field of view of only $180^{\circ}$ may be used and a complete electron distribution is obtained every spin period, i.e. 120 s. The ELECTRON detector provides a $180^{\circ} \times 6^{\circ}(\mathrm{FWHM})$ field of view with a uniform angular resolution of $22.5^{\circ} \times 8^{\circ}$.
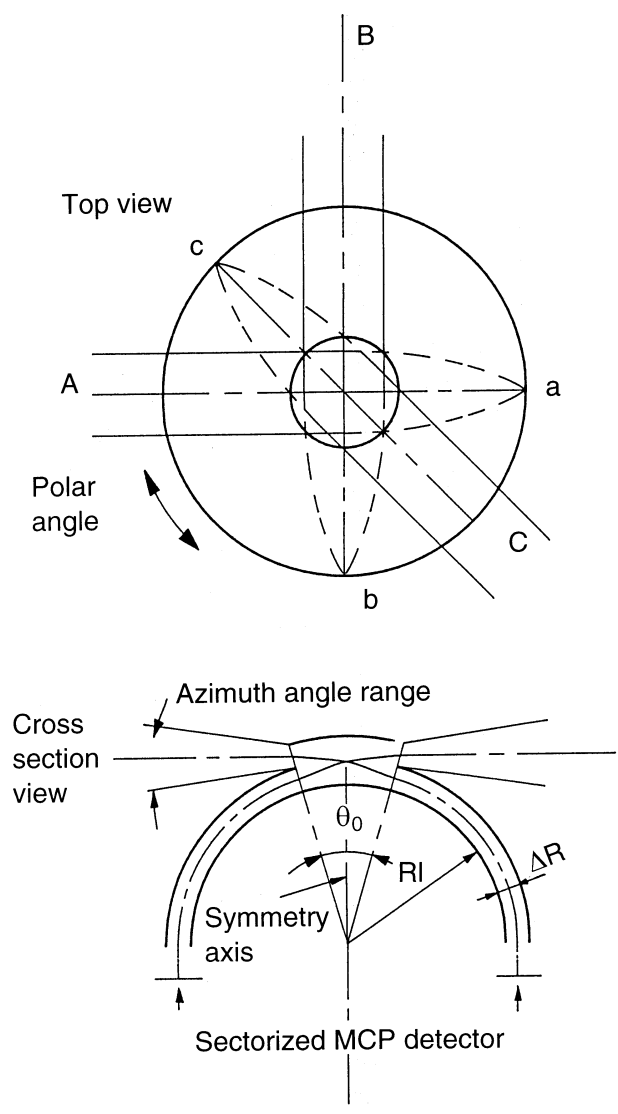

Fig. 1. Schematic views of the top-hat analyser and examples of parallel beam trajectories 


\subsection{Electron multipliers}

A schematic view of the microchannel plates (MCP) located at the output of the analyser and of the sectored collector is shown in Fig. 2. The Mullard MCP is made of two rings, each $1 \mathrm{~mm}$ thick. Each ring has a Ni-Cr metal coating on the two-face active surface. The total resistance across a ring has a low value for fast counting purposes. The $12.5-\mu \mathrm{m}$ diameter straight microchannels are inclined by $15^{\circ}$ and the two rings are chevronmounted with an inter-MCP gap of $30 \mu \mathrm{m}$. The collector associated with the MCP is divided into eight sectors of $22.5^{\circ}$. The $4 \pi$ analysis using the rotation of the spacecraft is usually done with $22.5^{\circ} \times 11.25^{\circ}$ or $22.5^{\circ} \times 22.5^{\circ}$ elementary sectors, except in special modes of operation when the measurement speed is 8 or 16 times higher.

\subsection{Sensor electronics and operating modes}

Signals from the eight MCP sectors are sent through charge-sensitive amplifiers/discriminators to eight counters. The energy selection of the particles is made by varying the deflection voltage of the inner plate of the electrostatic analyser between $\sim 1$ and $2600 \mathrm{~V}$. The variation of the deflection voltage can either be synchronized with the spin period of the spacecraft or free running. The deflection voltage is swept according to an exponential function. The sweep consists of 128 small steps to give an effectively continuous sweep. The counter accumulation time defines 32, 16, 4 or 2 energy intervals per sweep. The number of sweeps in a spin and the sweeping energy range can be adjusted according to the mode of operation. The instrument data system controls the operation of the analyser, formats the data for the telemetry channel and receives and executes commands. The processor is a $80 \mathrm{C} 86$ 16-bit microprocessor, together with RAM and ROM memories, and a "watch-dog" timer. The watch-dog timer is used to monitor the health of the software and reset the processor if the operation is abnormal. Sixteen different modes of operation can be chosen from the ground.

\section{Detection section}

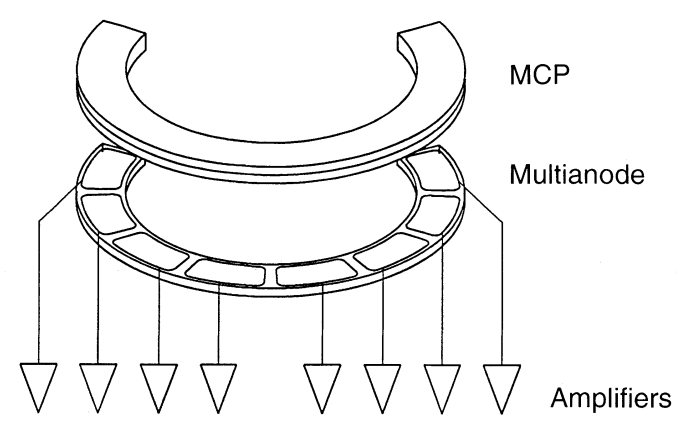

Fig. 2. Schematic view of the MCPs located at the output of the analyser and of the sectored anode which allows the discrimination of eight directions covering $180^{\circ}$
Generally 16 or 32 energy spectra with 16 or 32 logarithmically spaced energy steps are obtained during a spin, thus dividing the velocity-solid angle space into 8192 or 2048 elementary cells. Moment computations can be performed on board, from the density to the heat-flux vector, in order to reduce the necessary telemetry allocation. However, whenever possible, complete information is sent to ground. In special fast modes, priority is given to the measurement speed. A subset of four (resp. two) energies can be scanned every 240 (resp. 120) ms.

\subsection{Energy and angular resolutions}

The electron spectrometer was calibrated in the CESR vacuum facilities using a large electron beam $(\sim 200$ $\mathrm{cm}^{2}$ ). The analyser has an energy resolution (FWHM) of $\sim 12 \%$ which does not depend on the anode, i.e. it is independent of the polar angle (Fig. 1). The azimuthal angular resolution is $\sim 5^{\circ}$; the resolution in polar angle is $22.5^{\circ}$. The analyser constant $k=E / V$, where $E$ is the energy of the particle selected and $V$ the positive voltage applied to the inner hemisphere, varies slightly between $\sim 9.6$ and $9.8(\sim 2 \%)$ as a function of the polar angle. The uniformity of the MCP response has also been checked and estimated to be of the order of $\sim 4 \%$.

The energy-geometry factor of each of the eight sectors of the analyser $G$ expressed in $\mathrm{cm}^{2}$.ster.keV is equal to $7.7 \times 10^{-5} \times \mathrm{E}(\mathrm{keV})$. Colour energy-time spectrograms are given throughout the paper in units of counts/s. These values must be divided by $7.7 \times 10^{-5}$ to obtain the energy flux expressed in $\mathrm{keV} /\left(\mathrm{cm}^{2}\right.$.s.ster.keV).

\section{First results on the LLBL}

During the first two months following its launch, the INTERBALL-Tail spacecraft crossed the magnetopause and LLBL in the dawn sector at local times ranging from 07 to $03 \mathrm{~h}$. Inside this LT sector, the magnetopause was detected at radial distances of the order of 14 to 18 $R_{E}$ depending on the solar-wind pressure. The satellite crossed the magnetospheric equatorial plane at a radial distance of about 5 to $10 R_{E}$. The outer dawn magnetosphere was swept at GSM-Z coordinate between approximately 0 and $5 R_{E}$., i.e. at latitudes ranging from $\sim 0^{\circ}$ to $\sim 25^{\circ}$.

\subsection{Large-scale structure}

Figure 3 illustrates an energy-time spectrogram for electrons in the energy range $10-26000 \mathrm{eV}$ taken from 01:00 to 07:00 UT on 2 September 1995. The satellite was inside the magnetosheath until about 01:46 UT, when it crossed the magnetopause for the first time. Note that high-energy electrons were already detected in the magnetosheath on that day. Due to the satellite rotation these unidirectional field-aligned electrons appear in the figure as vertical bars between 01:00:00 


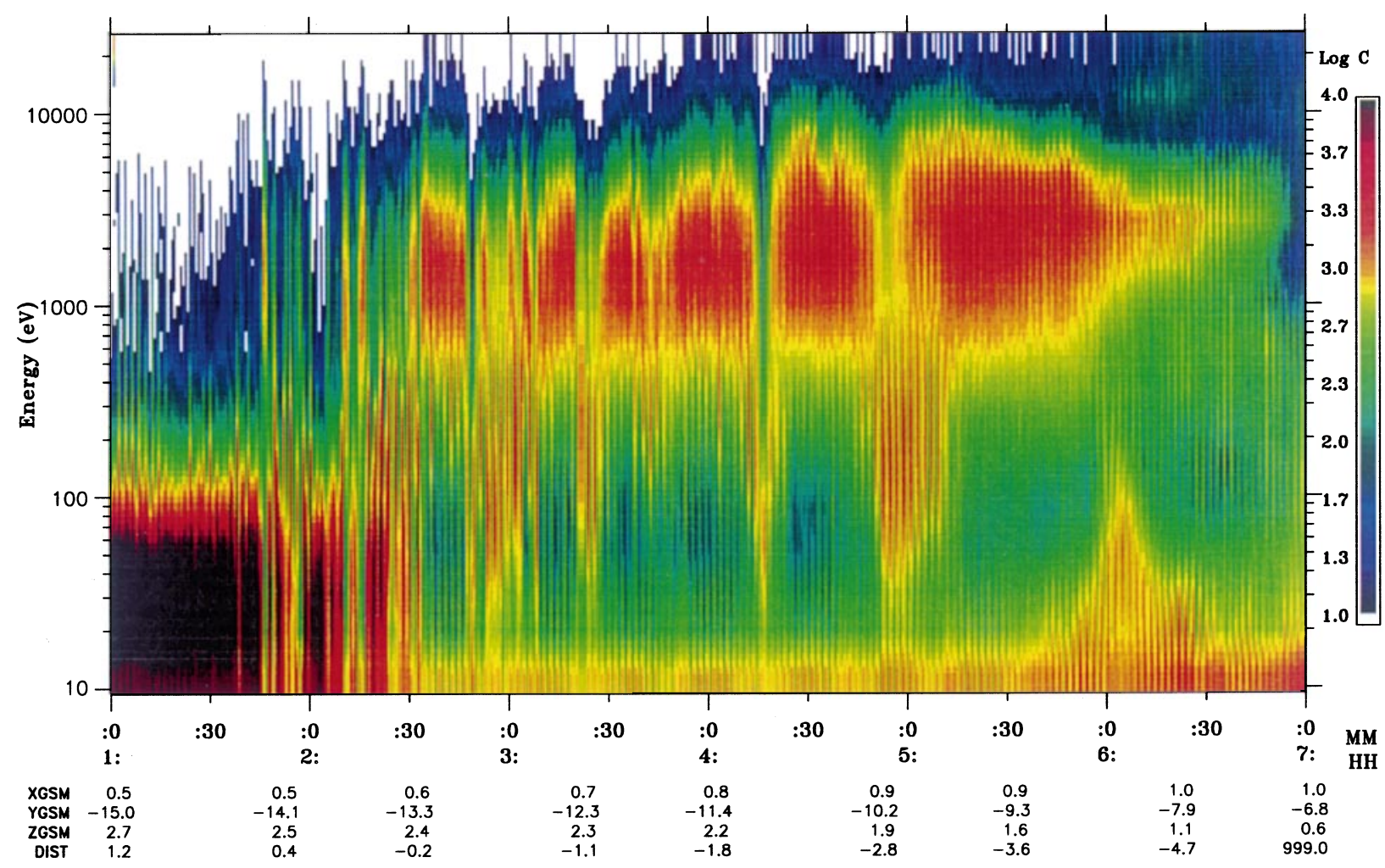

Fig. 3. Energy-time spectrogram of electrons in the energy range 10 $26000 \mathrm{eV}$ on 2 September 1995 between 01:00:00 and 07:00:00 UT. The data are delivered from the anode of the detector spinning in a plane perpendicular to the ecliptic. Inside the magnetosphere, the

and 01:30:00 UT. The interplanetary magnetic field was southward directed at that time. This observation, which strongly suggests a leakage of magnetospheric electrons along reconnected field lines, will briefly be presented later in this paper but deserves further study. After multiple magnetopause crossings, the satellite finally entered the magnetosphere at around 02:30 UT. From that time on, two plasma regimes were intermittently detected from the magnetopause at $(X, Y, Z)_{\mathrm{GSM}}=$ $(0.6,-13.7,2.5)$ up to the inner magnetosphere at $(X, Y$, $Z)_{\mathrm{GSM}}=(1.0,-7.0,1,0):$ (1) a high-energy plasma regime with an average energy of about $2 \mathrm{keV}$ and (2) a low-energy plasma, $E<1000 \mathrm{eV}$, whose occurrence, as a trend, is anti-correlated with the appearance of higherenergy electrons. Note that, finally, and closest to Earth, a third plasma domain is encountered, namely a region with very low energy plasma $(E<200 \mathrm{eV})$ which coincided with the disappearance of the high-energy electron population (05:50-06:20 UT). A more detailed examination of this low-energy component shows that the electrons here are field aligned for energies between 10 and $50 \mathrm{eV}$, and indicates the formation of loss-cones at energies of about $80 \mathrm{eV}$ and higher. This inner magnetospheric region will not be studied here in more detail. Inside the magnetosphere an electron component with energies lower than $15-20 \mathrm{eV}$ is measured. These non-gyrotropic electrons are photoelectrons produced magnetic field is roughly parallel to the $Z_{\mathrm{GSM}^{-}}$axis, so that the pitchangle range $\sim 0^{\circ}-180^{\circ}$ is scanned every minute due to the satellite rotation. On the left-hand side of the figure, the dense and low-energy magnetosheath plasma is seen as a black area on the spectrogram

by UV photons striking the satellite structure, which reaches a potential of about $10 \mathrm{~V}$.

It must be stressed that plasma region 1 is detected with an apparent period which increases from the magnetopause to the inner magnetosphere, from about 5 to $20 \mathrm{~min}$. Striations in the energy-time spectrogram are due to the satellite rotation. The data displayed are delivered by the anode, which is spinning in a plane nearly perpendicular to the ecliptic and contains the magnetic-field direction each minute, which is nearly aligned with the GSM-Z axis. The 1-min striations are indicative of field-aligned electrons, as we will later show in more detail. Note that these low-energy electrons are measured inside a region which extends over about $4 R_{E}$ from $Y_{\mathrm{GSM}}=13-14$ to $Y_{\mathrm{GSM}}=9.7 R_{E}$.

Figure 4 illustrates another dawn LLBL crossing, later in September when the satellite orbit had drifted towards the night-side by about $1 \mathrm{~h}(07 \mathrm{~h} \mathrm{LT})$. On 17 September (Fig. 4), the same general structures as in Fig. 3 can be recognized. Until about 04:20 UT the satellite was in the magnetosheath (with multiple magnetopause crossings before that time). After 04:20 UT, up to about 08:30 UT, the satellite encountered a highenergy electron component and sporadically high fluxes of low-energy electrons. There is an anti-correlation, as in the preceding crossing, between the detection of high fluxes of energetic electrons ( 1 to $10 \mathrm{keV}$ ) and that of the 
17/Sep/95 03:00:00

INTERBALL/ELECTRON

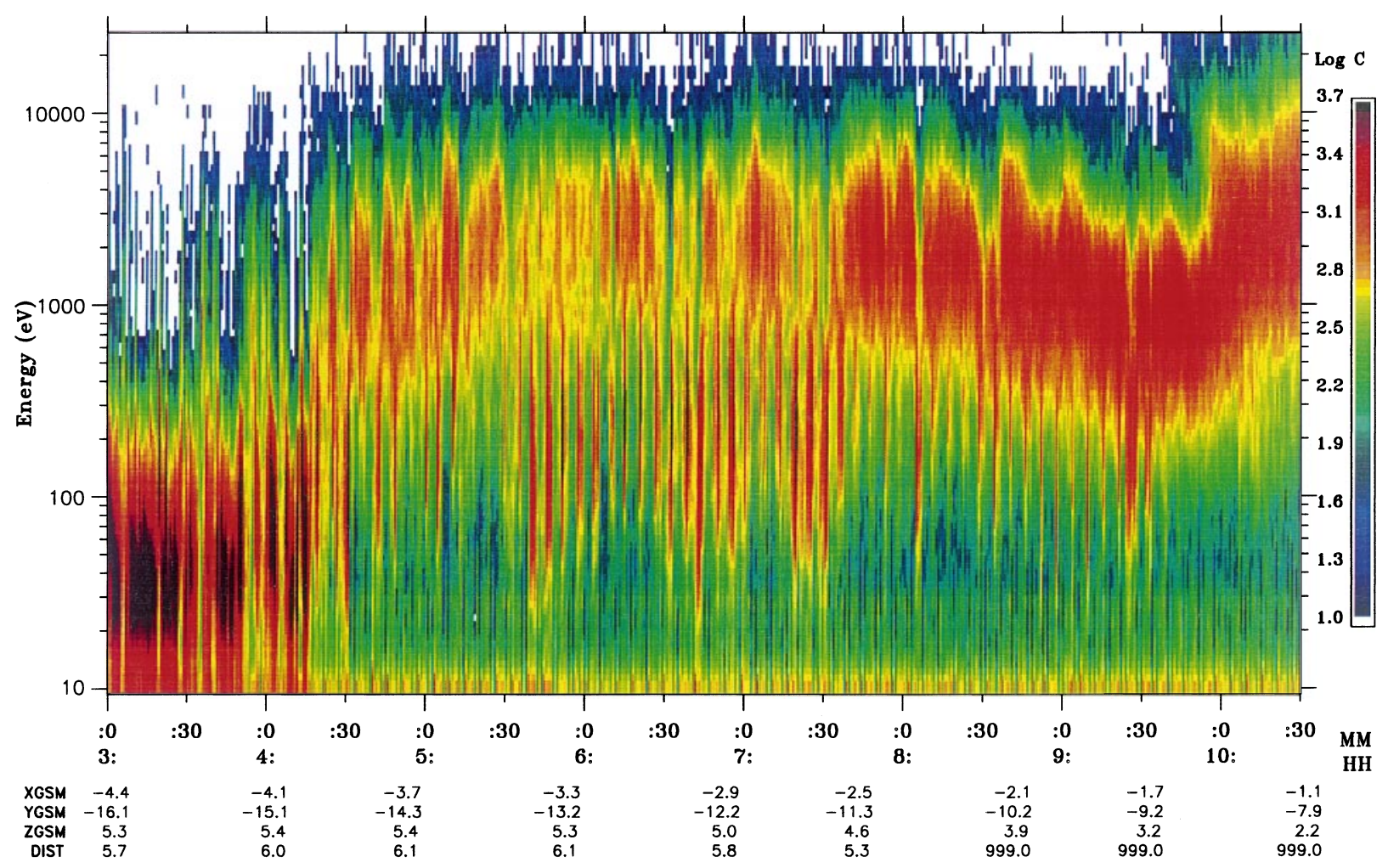

Fig. 4. Same as Fig. 3 for 17 September 1995, between 03:00:00 and 10:00:00 UT

17/Sep/95 01:30:00

INTERBALL/ELECTRON

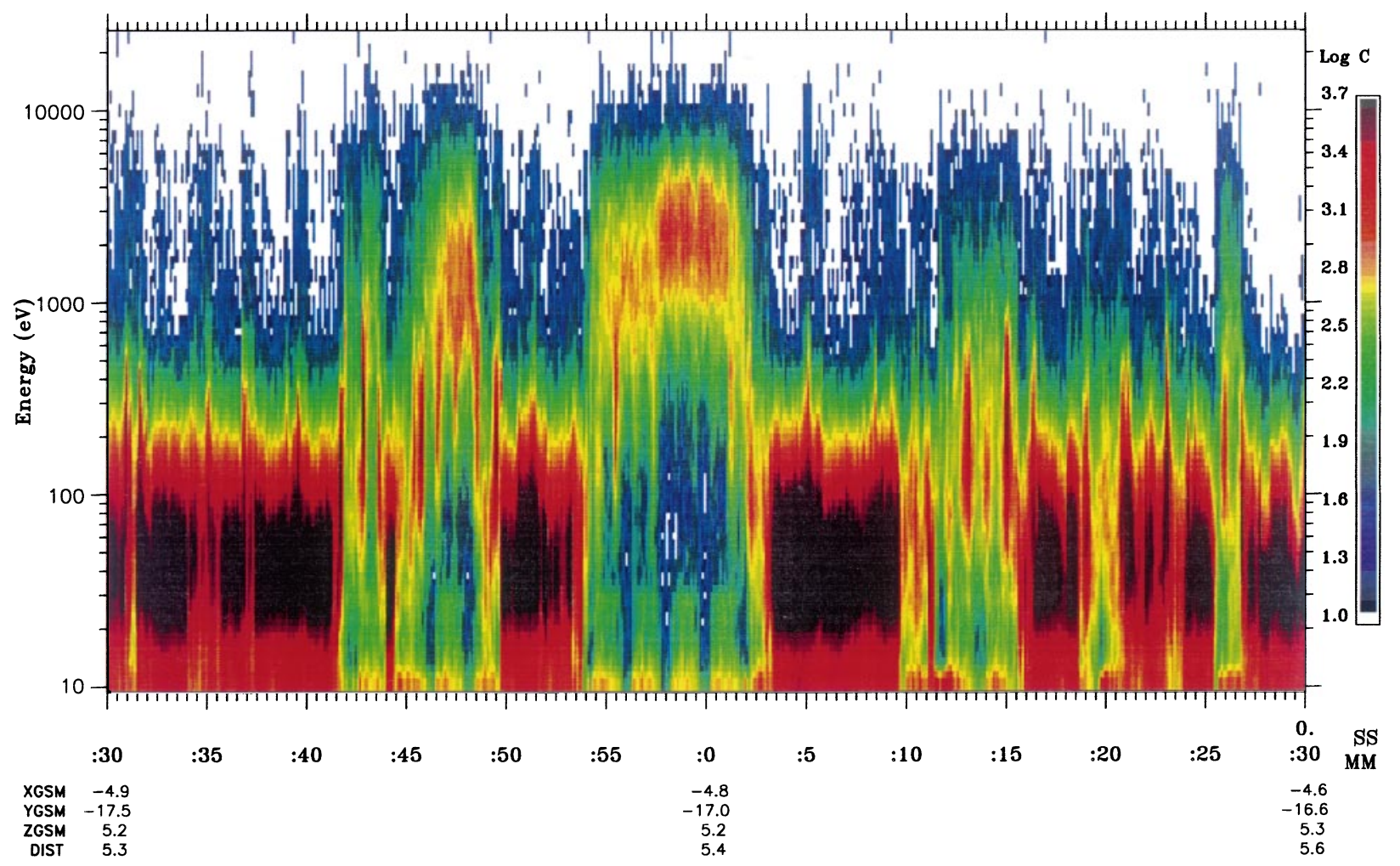

Fig. 5. Same as Fig. 3 for the time-period 01:30 to 02:30 on 17 September 1995, when the satellite encountered the magnetopause many times 
highest fluxes of low-energy electrons $(E<1 \mathrm{keV})$. Finally, at $\sim 09: 30-09: 40$ UT, the satellite detected drifting and energy-dispersed $\mathrm{keV}$ electron fluxes associated with a substorm seen at 09:30 UT on ground stations (GEM event). Note that the high-energy electron component showed a tendency after 06:00 UT towards an average energy decrease which could be attributed to the effect of electron drift from the nightside plasma sheet. Higher-energy electrons are first deflected by the gradient drift as they approach the Earth and thus drift at greater radial distances than the lower-energy electrons which can enter closer to the Earth before being energized and deflected.

From Figs. 3 and 4 two important conclusions can be reached: plasma-sheet electrons are detected from the magnetopause up to the inner magnetosphere, and the LLBL is sporadically encountered from the magnetopause position and extends, unexpectedly, very far inside the magnetosphere. As a trend, the detection of LLBL electrons occurs in coincidence with a decrease in the flux of the plasma-sheet electrons. However, the plasma sheet and LLBL can also be mixed, for example between 07:00 and 07:15 UT on 17 September (Fig. 4).

These conclusions are reinforced by Fig. 5, which presents observations also made on 17 September 1995 when the satellite was sporadically encountering the
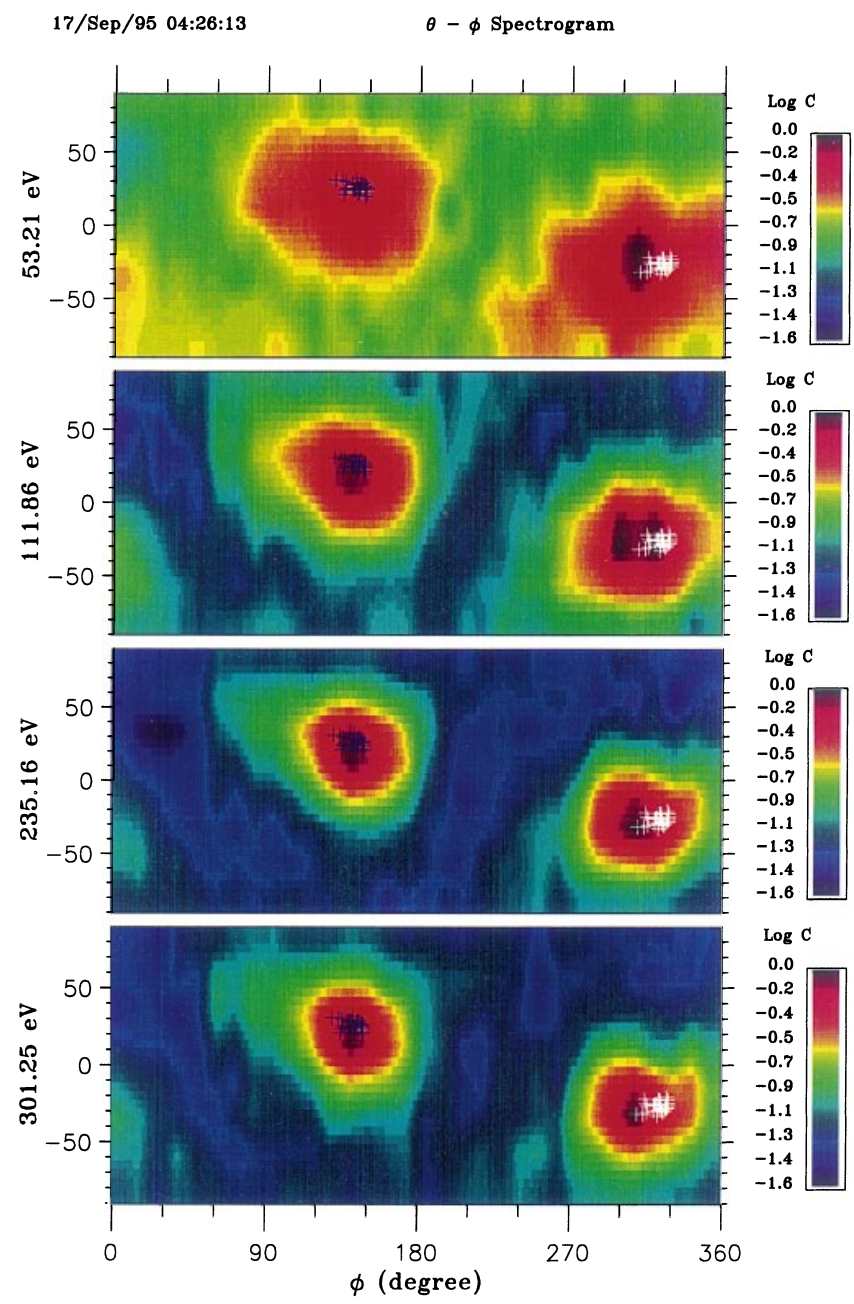

magnetopause between 01:30 and 02:30 UT, i.e. before the time-interval corresponding to Fig. 4. The magnetosheath appears here with maximum (black) fluxes around $40 \mathrm{eV}$. Plasma-sheet fluxes are detected in close association with the satellite entrance into the magnetosphere, for example between 01:45 and 01:50 UT and between 01:53:30 and 02:02:30 UT. LLBL electrons are present during the entire period at the magnetosheath/magnetosphere interface. A detailed study of this event, driven by the southward IMF, will be presented elsewhere.

\subsection{Electron distribution in the $L L B L$}

LLBL electron distributions over the unfolded $4 \pi$ solid angle corresponding to the time-interval 04:26:1304:28:13 UT on 17 September 1995 presented in Fig. 4 are given in Fig. 6 for four energies between 53.12 and $301.25 \mathrm{eV}$. It must be stressed that this period corresponds to the encounter of the LLBL electrons with very weak fluxes of plasma-sheet electrons. The vertical axis is the azimuthal angle (Fig. 1), with $+90^{\circ}$ being directed sunwards, $-90^{\circ}$ anti-sunwards, while the angle $\phi$ is the rotation angle. On each graph, white crosses indicate the direction of the $-B$ vector, and blue crosses the direction of the $+B$ vector. A spin period of 2 min is needed to sweep the total solid angle. During that time the magnetic-field direction varied slightly, which explains the dispersion of the crosses. Electrons are aligned at $0^{\circ}$ and $180^{\circ}$ with the magnetic field. This is a characteristic of the LLBL electrons, already found in the work of Ogilvie et al. (1984). Note that the field alignment is stronger as the energy increases. The flux anisotropy ratio $J\left(\alpha \sim 0^{\circ}\right) / J\left(\alpha \sim 90^{\circ}\right)$ reaches a factor of $\sim 10$ at $110-180 \mathrm{eV}$. The electron temperature parallel to the magnetic field is of the order of $100 \mathrm{eV}$.

Figure 7 gives the electron distribution at 04:40:15 UT, i.e. when the satellite is measuring both LLBL electrons and plasma-sheet electrons. Here the lowenergy electrons are field-aligned, counterstreaming as expected inside the LLBL. The electron distribution is nearly isotropic at medium energies $(\sim 800 \mathrm{eV})$ and displays a loss-cone distribution at higher energies $(\sim 2000 \mathrm{eV})$. It must be stressed that a loss-cone distribution is expected for electrons drifting from the nightside plasma sheet to the dawnside under the regime of weak pitch-angle diffusion. This reinforces the identification of the high-energy electron population as plasma-sheet electrons drifting to the dawnside under the conjugate effects of the magnetic gradient and electric drifts. For comparison, in Fig. 8, we show the

Fig. 6. $4 \pi$ electron distributions at four selected energies between 53.21 and $301.25 \mathrm{eV}$ in the LLBL on 17 September 1995, from 04:26:13 to 04:28:13 UT. The vertical axis gives the polar angle varying from the solar direction $\left(+90^{\circ}\right)$ to the anti-solar direction $\left(-90^{\circ}\right)$; the horizontal axis is the rotation angle. The satellite spin axis is pointing towards the Sun and the $180^{\circ}$ detector field of view contains the spin axis. The blue crosses indicate the magnetic-field direction, the white crosses the direction anti-parallel to the magnetic field 

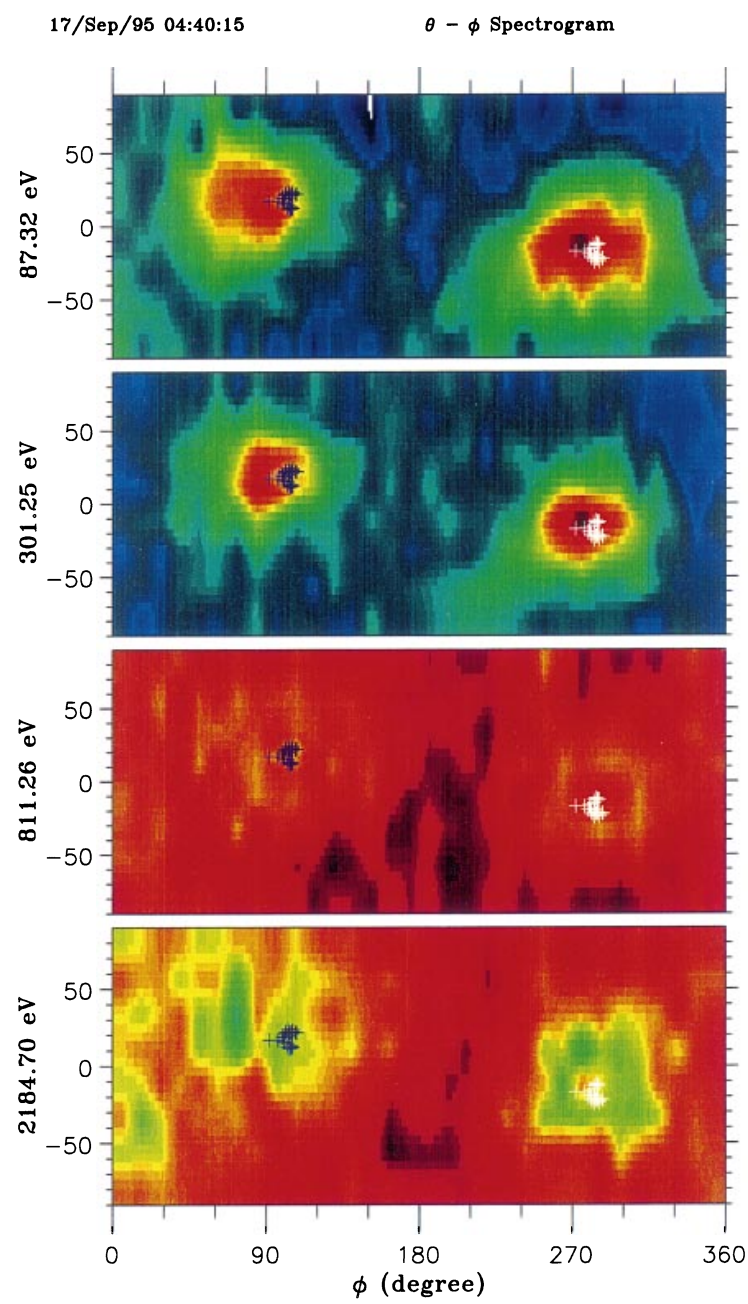

Fig. 7. Same as Fig. 6 but for a region where the LLBL and plasmasheet plasma are mixed. Note the appearance of a loss-cone at the highest energies

electron distribution measured in the magnetosheath on 2 September 1995 at 01:01:28 (Fig. 4). At the lowest energy, $\sim 32 \mathrm{eV}$, the magnetosheath electrons are nearly isotropic with a slight flux depression along the magnetic field. At higher energies, up to several $\mathrm{keV}$, the electrons display a unidirectional beam directed along the field line from the magnetopause direction. This experimental evidence strongly suggests a leakage of magnetospheric electrons through the magnetopause.

\subsection{Relationship between the LLBL and magnetic-field structures}

Initial results of simultaneous measurements of electrons and magnetic fields are displayed in Fig. 9 for 2 September 1995. Here the electron flux perpendicular to the magnetic field has been plotted for two energies: 100 and $5000 \mathrm{eV}$. At the top of the figure the three components $B_{z}, B_{x}$ and $B_{y}$ are given in the GSM frame. The total magnetic field is nearly equal to its $B_{z}$ component. The appearance of LLBL electrons and

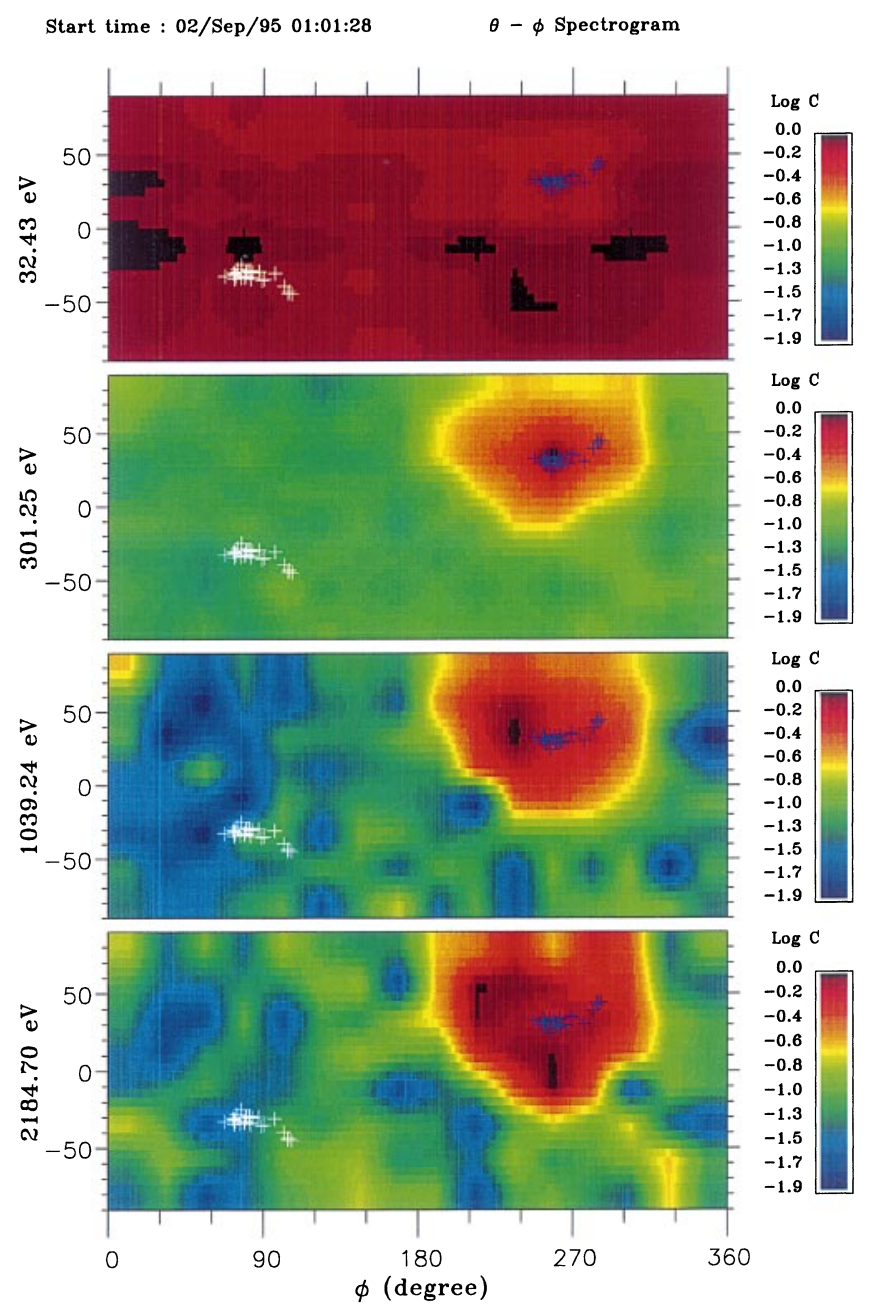

Fig. 8. Same as Fig. 6 but for unidirectional beams encountered in the magnetosheath on 2 September 1995

the associated strong decrease in plasma-sheet electron fluxes clearly coincides with a strong increase in the magnetic field between 04:14 and 04:26:40 UT. During the same period the ion measurements from the SKA-1 experiment display a low-temperature ion component and the quasi-disappearance of plasma-sheet ions (O. Vaisberg, private communication, 1996), so that the increase in the $B$ field can be interpreted as being due to the conservation of the total pressure. Before the LLBL encounter, the $B_{y}$ component of the magnetic field was negative, indicating that the satellite was located under the magnetospheric equatorial plane. The detection of the LLBL cloud is done in two main steps. Between 04:08 and 04:14 UT, an initial decrease in the plasma-sheet electrons is associated with a negative excursion of the $B_{y}$ component and with a positive excursion of the $B_{x}$ component which could be attributed to the effect of a field-aligned current structure moving Earthwards and tailwards, the current going to the southern ionosphere (region-1 current). Note that during this event the total magnetic field was only slightly enhanced. The second step began at 04:14 UT with a much steeper decrease in plasma-sheet electron 


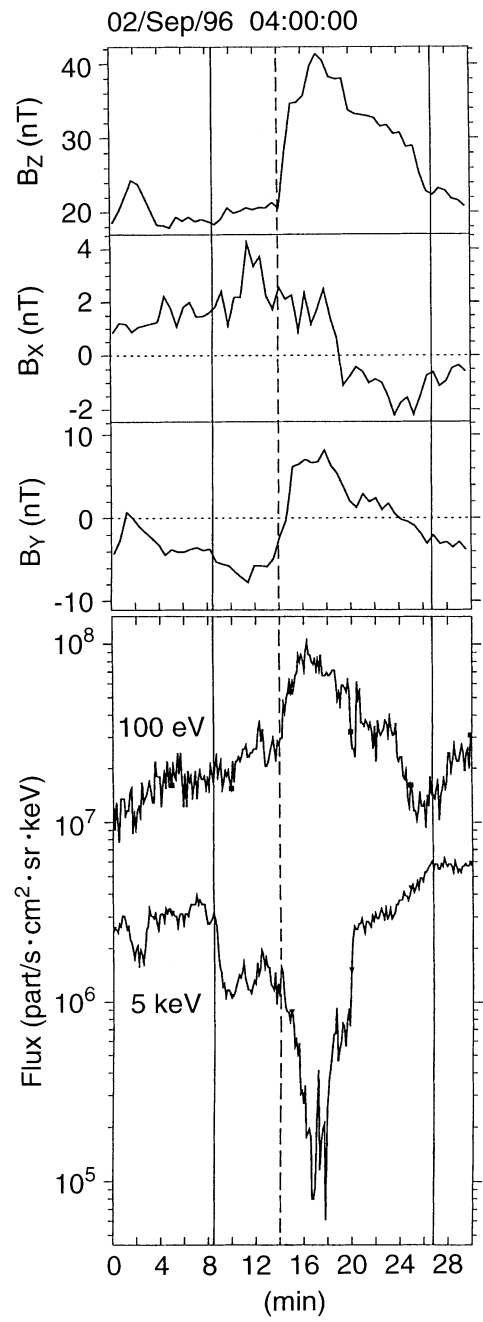

Fig. 9. From top to bottom, the magnetic-field components, the total $B$ field and the 100 and $5000-\mathrm{eV}$ electron fluxes for 2 September 1995 from 04:00:00 and 04:30:00 UT. The LLBL corresponds to high fluxes of low-energy electron and strong decrease in the $\mathrm{keV}$ electron fluxes. Note the strong increase in the $B$ field in the LLBL cloud and the bipolar variation of the $B_{x}$ component of the magnetic field between 04:14:00 and 0426:40 UT (see text)

flux. The magnetic field reached $42 \mathrm{nT}$ at 04:18 UT, i.e. twice its pre-event value. The $B_{x}$ component displayed a reversal, first positive, then negative, while the $B_{y}$ component displayed a unipolar variation. Again these variations are in agreement with the encounter of a fieldaligned structure with the same current direction as in the preceding case. However, in order to explain the observations, the velocity of the current structure has to be more Earthwards-directed. These preliminary results are in agreement with the LLBL as the source of region1 currents (Eastman et al., 1976; Lundin and Evans, 1985). It must also be stressed that due to the strong magnetic field inside the LLBL clouds and the predominance of gradient drift for high-energy particles, we can expect the plasma-sheet particles not to penetrate inside LLBL clouds, as indeed observed.

\section{Discussion and conclusion}

The preliminary results obtained from the electron experiment aboard the INTERBALL-Tail spacecraft show that LLBL electrons are observed inside an unexpectedly large region of the dawnside of the magnetosphere. LLBL electrons associated with high $B$ fields can be seen many Earth radii inside the position of the magnetopause encountered on the same orbit. The location and extension of the region in the equatorial GSM plane along the INTERBALL orbits where LLBL electrons have been detected are given in Fig. 10. The fact that the $B_{z}$ component of the magnetic field is always positive in that region, and that bi-directional low-energy field-aligned and loss-cone distributions of $\mathrm{keV}$ electrons are simultaneously detected, strongly suggest that LLBL electrons are located inside closed magnetospheric field lines.

It must be stressed that inside the inner LLBL the magnetopause is generally no longer encountered, which indicates that the real width of the LLBL is large, even if this region is subjected to wavy deformations. One can speculate that the intermittent appearance of low-energy LLBL electron clouds and the associated quasi-disappearance of the plasma-sheet electrons could indicate the action of surface waves such as the Kelvin-Helmholtz instability resulting from the anti-parallel flows inside the LLBL and inside the plasma sheet. However, such an interpretation requires further testing. Information can be deduced using simultaneous data obtained aboard the INTERBALL-Tail subsatellite, MAGION 4, located ahead of INTERBALL-Tail, on the same orbit and 13 min away. Also, the correlation between the LLBL width and solar wind or auroral activity could help to characterize the process responsible for the formation

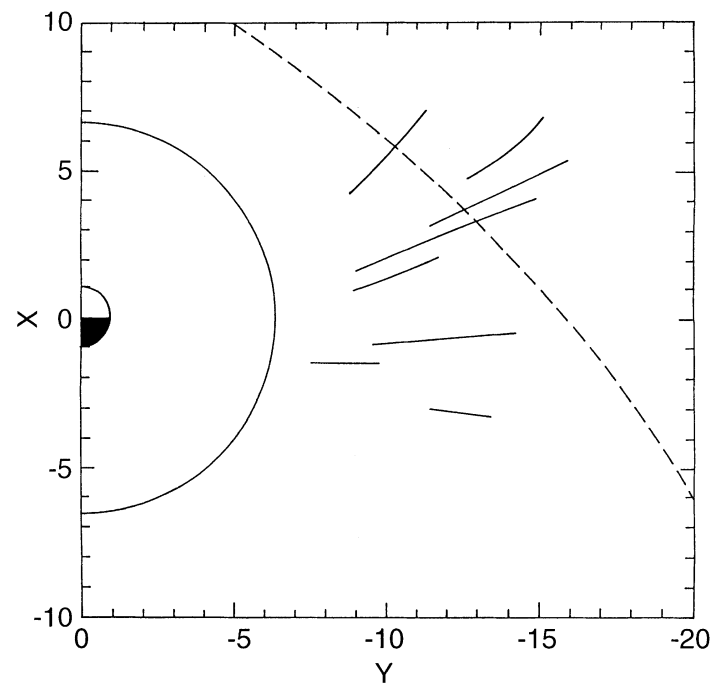

Fig. 10. Distribution in the GSM equatorial plane of the portions of the INTERBALL orbit where LLBL was encountered from the end of August to the end of September 1995. The orbital period is approximately 4 days, so that the satellite orbit has drifted by about $4^{\circ}$ between two consecutive inbound passes in the dawn sector. A geostationnary orbit and a simple model magnetopause are shown for reference 
of such large scale structure. For all INTERBALL passes, WIND measurements of magnetic-field and plasma parameters have been examined [see Lepping et al. (1995) and Lin et al. (1995) for experiment descriptions]. Also the energy coupling function between the solar wind and the magnetosphere has been estimated (Akasofu, 1981). The only trend which has been found is that large LLBL are seen for high solar-wind density and large positive $B_{Z}$ (averaged over the satellite pass in the LLBL). It must be stressed that the increase in LLBL width for positive $B_{z}$ has already been reported by Mitchell et al. (1987). Finally, one should note that the unexpected large dimensions of the LLBL reinforce the conclusion of numerous measurements showing a high rate of entry of solar wind plasma into the magnetosphere through the LLBL (e.g. Lundin et al., 1991).

Acknowledgments. The construction of the ELECTRON experiment was supported by the French space agency, CNES, under Contract No. CNES-208. We are grateful to J.-P. Thouvenin, D. Fournier and G. Azoulay for their support and encouragement throughout the entire INTERBALL project. The support of the Russian project team during the experiment testing and integration on the INTERBALL-Tail satellite was greatly appreciated; we would particularly like to thank this team for making the INTERBALL-Tail satellite a reality. Data processing has been made possible by E. Penou, J. Durand and their colleagues. The authors thank M. Nozdrachev and S. Romanov PI's of the FM3-I and MIF-M magnetometers, respectively, who have kindly provided the magnetic-field data used in this paper.

Topical Editor K. -H. Glaßmeier thanks C- J. Owen and another referee for their help in evaluating this paper.

\section{References}

Akasofu, S.-I., Energy coupling between the solar wind and the magnetosphere, Space Sci. Rev., 28, 121, 1981.

Carlson, C. W., D. W. Curtis, G. Paschmann, and W. Michael, An instrument for rapidly measuring plasma distribution functions with high resolution, Adv. Space Res., 2, 67, 1982.

Eastman, T. E., E. W. Hones Jr., S. J. Bame, and J. R. Asbridge, The magnetospheric boundary layer: site of plasma momentum, and energy transfer from the magnetosheath into the magnetosphere, Geophys. Res. Lett., 3, 685, 1976.

Eastman, T. E., L. A. Frank, and C. Y. Huang, The boundary layer as the primary transport regions of the earth's magnetotail, $J$. Geophys. Res., 90, 9541, 1985.

Fuselier, S. A., B. J. Anderson, and T. G. Onsager, Particle signatures of magnetic topology at the magnetopause: AMPTE/ CC obersvations, D. Geophys. Res. 100, 11805, 1995.

Galeev, A. A., Yu. I. Galperin, and L. M. Zelenyi, The Interball project to study solar-terrestrial physics, in Interball mission and payload, ed. by RKA, IKI and CNES, Toulouse, pp. 11-32, 1995.

Gosling, J. T., J. R. Asbridge, S. J. Bame, and W. C. Feldman, Effects of a long entrance aperture upon the azimuthal response of spherical section electrostatic analyzers, Rev. Sci. Inst., 49, $1260,1978$.
Heikkila, W. J., Impulsive plasma transport through the magnetopause, Geophys. Res. Lett., 13, 233, 1986.

Lepping, R. P., M. H. Acuna, L. F. Burlaga, W. M. Farrell, J. A. Slavin, K. H. Schatten, F. Mariani, N. F. Ness, F. M. Neubauer, Y. C. Whang, J. B. Byrnes, R. S. Kennon, P. V. Panetta, J. Scheifele, and E. M. Worley, The WIND magnetic field investigation, Space Sci. Rev., 71, 207, 1995.

Lin, R. P., New techniques for charged particle measurements in the interplanetary medium, in Solar system plasma physics, Eds. J. H. Waite Jr., J. L. Burch, and R. L. Moore, AGU Geophys Monogr. 54, p. 333, 1989.

Lin, R. P., K. A. Sanderson, S. Ashford, C. Carlson, D. Curtis, R. Ergun, D. Larson, J. McFadden, M. McCarthy, G. K. Parks, H. Rème, J. M. Bosqued, J. Coutelier, F. Cotin, C. d'Uston, K.-P. Wenzel, T. R. Sanderson, J. Henrion, J. C. Ronnet, and G. Paschmann, A three-dimensional plasma and energetic particle investigation, Space Sci. Rev., 71, 79, 1995.

Lundin, R., and D. S. Evans, Boundary-layer plasmas as a source for high-latitude, early afternoon, auroral arcs, Planet. Space Sci., 33, 1389, 1985.

Lundin, R., I. Sandahl, J. Woch, and R. Elphinstone, The contribution of the boundary layer EMF to magnetospheric substorms, in Magnetospheric substorms, AGU Geophu Monogr. 64, Eds. J. R. Kan, T. A. Potemra, S. Kokubun, and T. Iijima, p. $355,1991$.

Mitchell, D. G., F. Kutchko, D. J. Williams, T. E. Eastman, L. A. Frank, and C. T. Russell, An extended study of the low-latitude boundary layer on the dawn and dusk flanks of the magnetosphere, J. Geophys. Res., 92, 7394, 1987.

Mozer, F. S., Electric field evidence on the viscous interaction at the magnetopause, Geophys. Res. Lett., 11, 135, 1984.

Ogilvie, K. W., R. J. Fitzenreiter, and J. D. Scudder, Observation of electron beams in the low-latitude boundary layer, J. Geophys. Res., 92, 7394, 1984

Paschmann, G., H. Loidl, P. Obermeyer, M. Ertl, R. Laborentz, N. Sckopke, W. Baumjohann, C. W. Carlson, and D. W. Curtis, The plasma instrument for AMPTE IRM, IEEE Trans. Geosci. Rem Sensing, GE-23, 262, 1985.

Rème H., F. Cotin, A. Cros, J. L. Medale, J.-A. Sauvaud, C. d'Uston, A. Korth, A. K. Richter, A. Loidl, K. A. Anderson, C. W. Carlson, D. W. Curtis, R. P. Lin, and, D. A. Mendis, The Giotto RPA-Cppernic plasma experiment, in ESA SP-1077, Ed. R. Reinhard and B. Battrick, ESA Publication Div., The Netherlands, p. 33, 1986.

Sauvaud, J.-A., H. Barthe, C. Aoustin, J. J. Thocaven, J. Rouzaud, J. Bouyssou, M. Cassignol, P. Souleille, J. M. Bosqued, T. Beutier, J. Crasnier, J. Dandouras, C. Jacquey, and

H. Rème, The electron experiment: a top-hat spectrometer for the tail probe, in INTERBALL mission and payload Ed. RKA, IKI and CNES, Toulouse, pp. 153-169, 1995.

Stasiewicz, K., New methods and techniques in visualization and mapping of magnetospheric boundaries, in Physical signatures of magnetospheric boundary layer processes, Eds. J. A. Holtet and A. Egeland, NATO ASI series, Kluwer, Dordrecht, pp. 433-447, 1994.

Traver, D. P., D. G. Mitchell, D. J., L. A. Frank, and C. Y. Huang, Two encounters with the flank low-latitude boundary layer: further evidence for closed field line topology and investigation on the internal structure, J. Geophys. Res., 96, 21025, 1991.

Tsyganenko, N. A., A magnetospheric magnetic field model with a warped tail current sheet, Planet. Space Sci., 37, 5, 1989. 